\title{
UMTS UPLINK SIMULATOR
}

This paper presents the link level simulator developed at the Telecommunication Department of the University of Žilina. The simulator was developed as part of the project VEGA No. 1/0140/03 "Effective radio resources management methods in next generations of mobile communication networks" and it will be used in the more complex radio interface simulator of the next generation of mobile communication network built at our department. Students studying some problems about spread spectrum methods will also use the simulation model in some subjects, e.g. Digital communications, Mobile Radio Networks.

The simulator was developed in MATLAB Simulink and is made on a block principle, which allows adapting and configuring the model as necessary.

In this contribution we present the model solution and some simulation results in an uplink direction. The intention of the contribution is not to present new and original results (similar simulators were previously developed in many institutions [1,2]), but to give an overview what will be realized at our department in the field of mobile communication networks.

\section{Introduction}

The UMTS (Universal Mobile Telecommunication System) is the third generation mobile communication system, which is scheduled to start service in Europe as a part of IMT-2000 (International Mobile Telecommunication). It is designed to provide a wide range of services to mobile and stationary users in a variety of working environments and application areas.

The UMTS system is based on two different solutions: WCDMA (Wideband Code Division Multiple Access), which uses frequency division duplex (FDD) and TD-CDMA (Time Division CDMA) designed for time division duplex (TDD). The presented simulation model is based on FDD mode [4].

The simulator is designed to evaluate the Bit Error Rate (BER) and Frame Error Rate (FER), which can be measured as a function of signal to interference ratio (SIR), spreading factor (SF), propagation channel ( 8 channel types), scrambling code, interleaver and Forward Error Correction (FEC) type.

Multiple access interference is generated by up to 8 interfering terminals with adjustable parameters.

\section{Description of the simulator}

The general structure of the radio chain is almost the same for voice and data services, so we used some simplification in the construction of time frames to be able to compare the results.

From a general point of view, the transmission part of the radio chain includes the following modules: source of binary data, CRC (Cyclic Redundancy Code) and tail bits insertion, channel coding, rate matching, interleaving, spreading, scrambling and modulation (Fig. 1) [1].

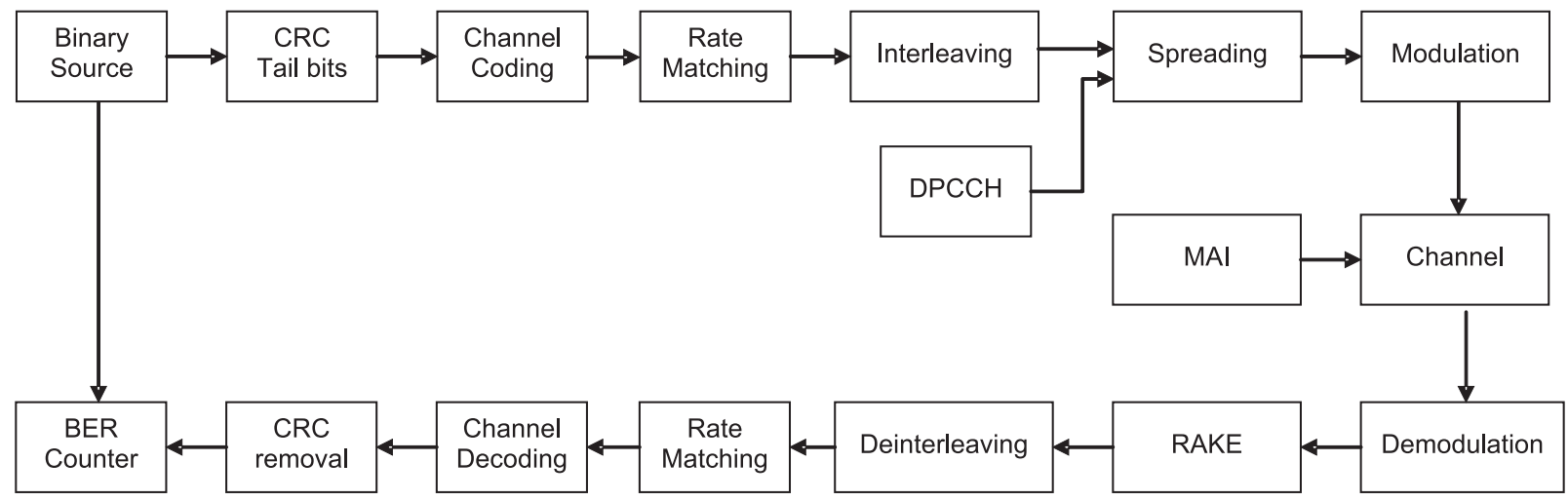

Fig. 1 Block diagram of the UMTS uplink radio chain

\footnotetext{
* Vladimír Wieser, Tomáš Marek

Telecommunication Department, University of Žilina, tel. 041513 2260, E-mail: wieser@fel.utc.sk, kauly@szm.sk, student
} 
The model blocks were created by 3 GPP and ETSI specifications $[5-9,12]$.

Because of a very complex uplink frame structure for different services (transmission rates) we used a simplified version designed for voice services and altered only a spreading factor, which defines the transmission rate used in the channel.

After inserting 16 bits of CRC code and 8 tail bits the stream is channel coded with convolution code of coding rate $1 / 2,1 / 3$ or none (code constraint $K=9$ ). Puncturing or repetition, to meet the closest rate for the chosen spreading factor SF does rate matching. The signal is interleaved (possibility to change the interleaver depth and the time, for which the maximum group error spans) and after multiplexing with a control channel DPCCH $(\mathrm{SF}=256)$ it is spread in a spreading modulator.

The spreading is realized in the same manner for desired and interfering users (Fig. 2). After multiplying data and control channel by different channelization codes (Orthogonal Variable Spreading Factor - OVSF Codes) the resultant summing signal is multiplied by the complex scrambling code (unique signature of the user).
The propagation channel is made up of a multipath-fading generator described in details in [3]. The propagation environments and models defined by ETSI and ITU-R [11] are:

1. Indoor / Low Range Outdoor - Office A, Office B, Outdoor to Indoor \& Pedestrian A.

2. Suburban Outdoor - Outdoor to Indoor \& Pedestrian A, Outdoor to Indoor \& Pedestrian B, Vehicular A.

3. Rural Outdoor - Vehicular A, Vehicular B.

These models are used in propagation simulation of useful and interference signals, respectively. The model allows switching off the short-term fading of each interfering source and setting up the propagation loss. It is also possible to model a propagation channel defined by the user who can set up a number of paths (up to six), delay and propagation loss for each path and speed of mobile terminal.

Multiple access interference (MAI) is modeled by up to eight interfering mobile terminals, which can be configured as it is shown in Fig.3.

The main window of the simulation model is in Fig. 4.

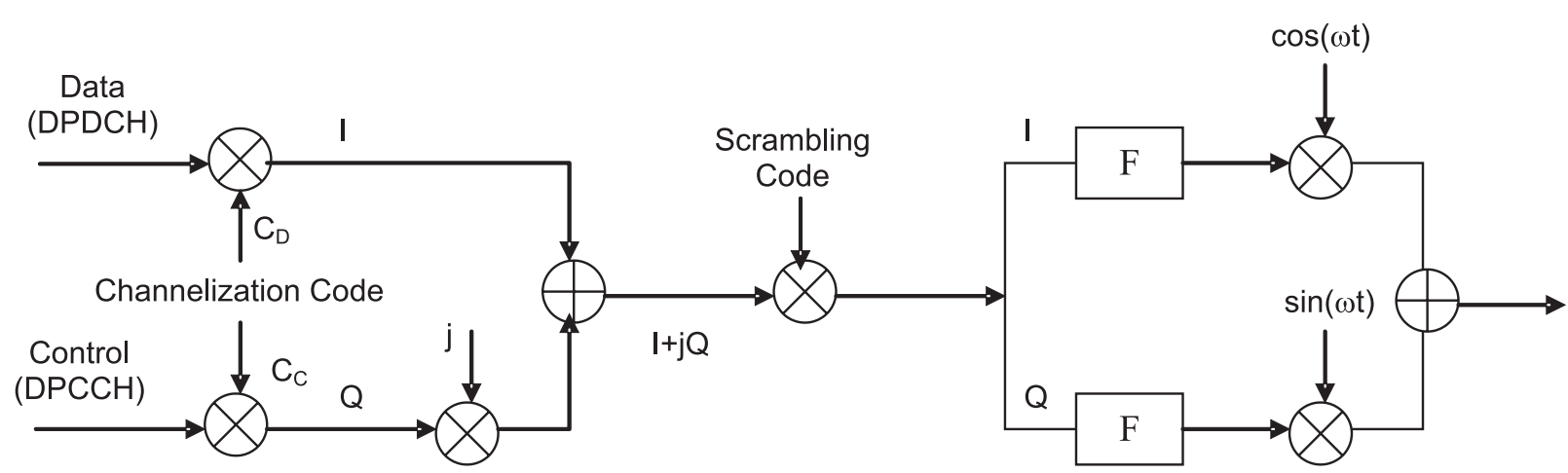

Fig.2 Uplink spreading and modulation

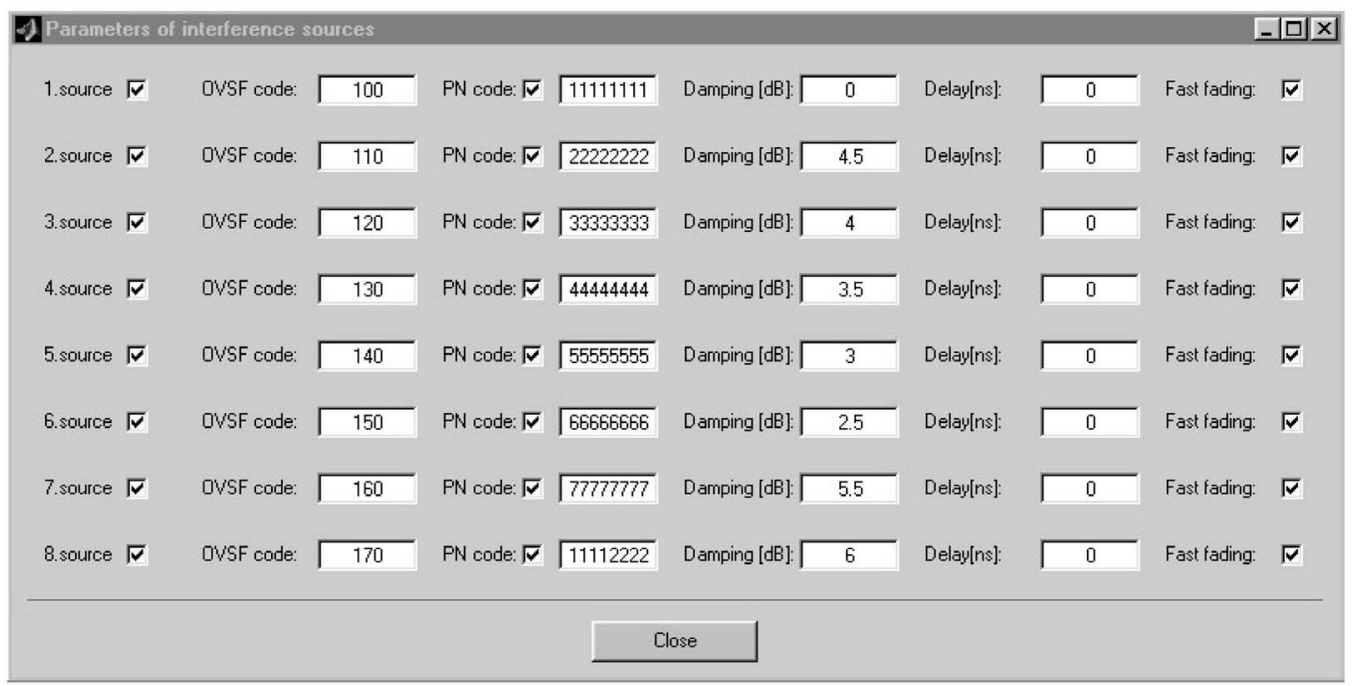

Fig. 3 Set up of interfering sources 


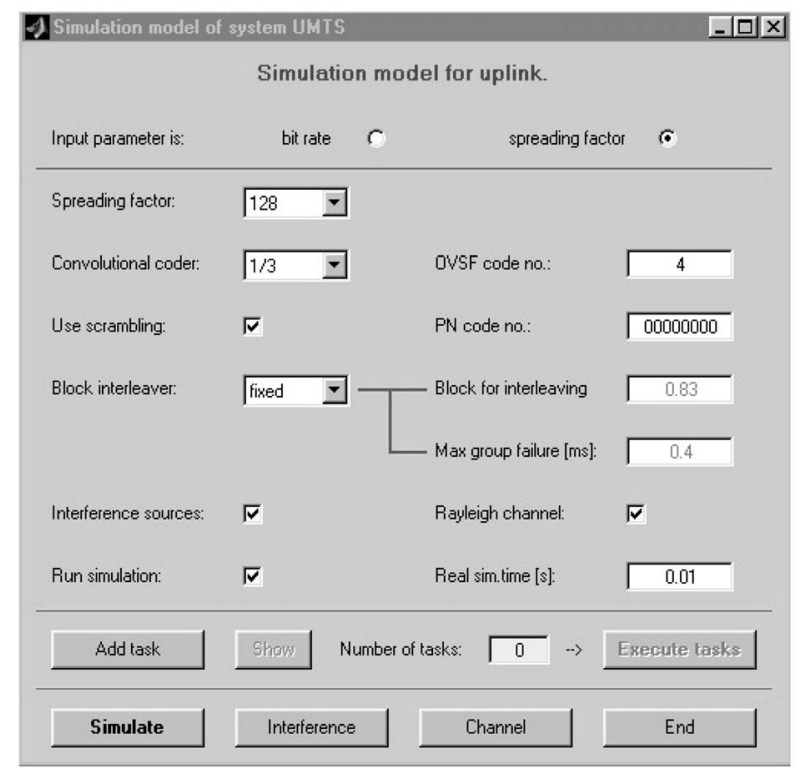

Fig.4 The main simulator's window

The receiving part consists of a Rake receiver with four branches tuned on the channel paths according to a chosen propagation model. De-interleaver and Viterbi decoder follows the Rake receiver, which is shown in Fig.5.

Unlike other Rake simulators [1,2] we implemented a possibility of switching off each of the Rake fingers, which gives us the option to simulate the situation with more paths than is the number of Rake fingers. This situation is the most probable one in real channel and environment with many reflectors in the surroundings of the receiver.

\section{Simulation results}

The UMTS system uses orthogonal OVSF codes for channelization purposes (spreading codes) and PN codes (Pseudo-noise) as scrambling ones. The intention of scrambling codes is to improve bad autocorrelation and cross-correlation functions (in the system without synchronization) of OVSF codes and to differentiate users in the uplink direction.

To show the system behavior in different situations we simulated the situation when the system uses the same OVSF codes in each neighbor cell.

The most inconvenient situation arises when all mobiles (useful and interfering) are situated on the cell boundary. We simulated three different dependences. In all simulations we used Rayleigh fading channel (Clarke's model).

Fig. 6 shows the dependence of BER on the number of interfering sources for three different situations. The output transmission power of the useful mobile and the interfering one are the same $\left(P_{\text {out } \_M S}=P_{\text {int }}\right)$.

The importance of scrambling codes using is obvious because of the cross-correlation among the same orthogonal OVSF codes. If the system uses the same scrambling code for each mobile, BER is worse than in the "normal" situation (each mobile uses its own

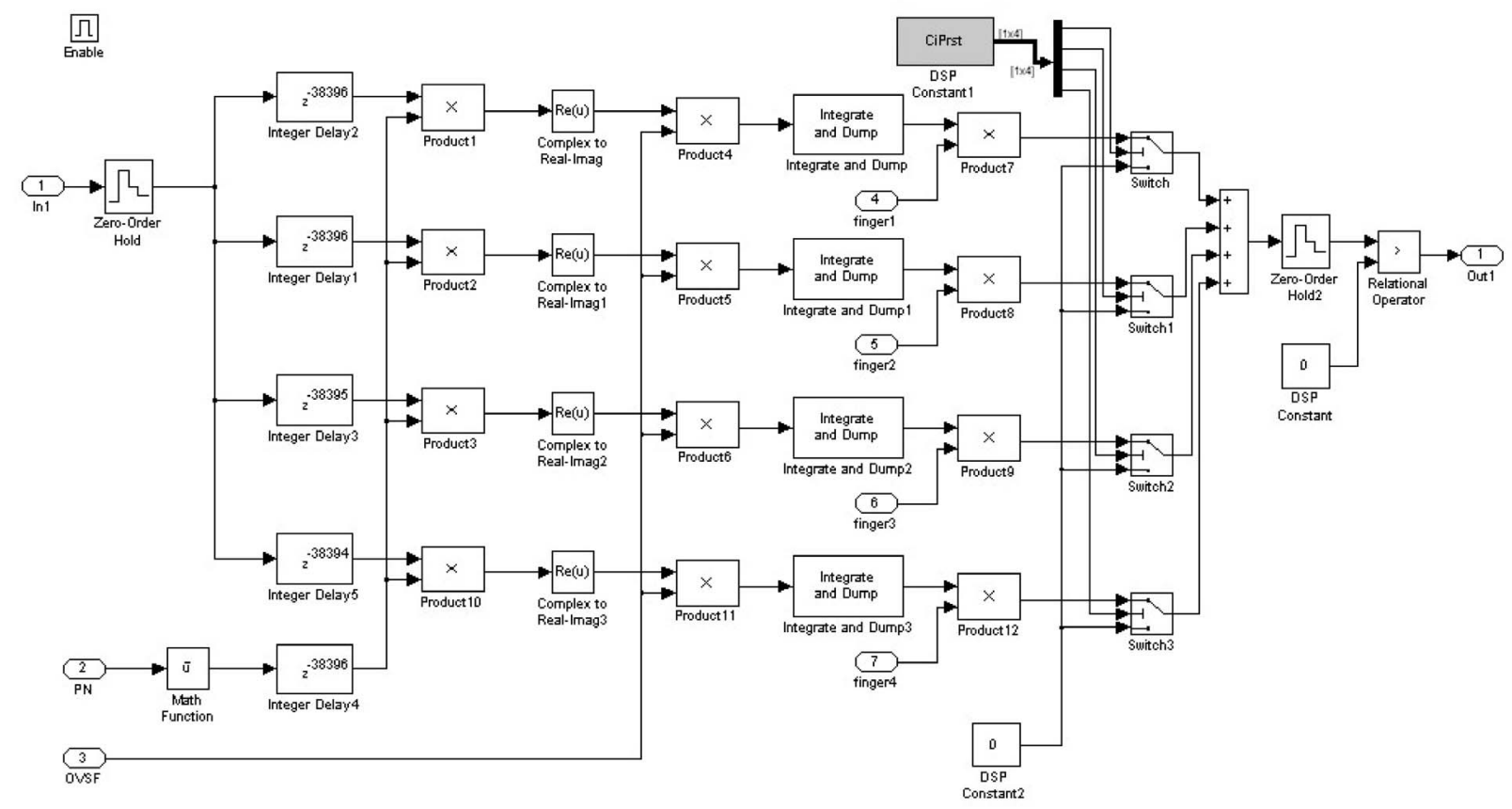

Fig. 5 Rake receiver 
scrambling code), but it is much better than in a situation when the system doesn't use scrambling codes at all.

The next simulation (Fig. 7a, b) shows the dependence of $\mathrm{BER}=f(\mathrm{SIR})$ and $\mathrm{FER}=f(\mathrm{SIR})$ for the spreading factor $\mathrm{SF}=4$ (all mobiles), scrambling codes are different and mobiles use convolutional coding with the code rate $\mathrm{R}=1 / 2, \mathrm{R}=1 / 3, \mathrm{R}=0$ (without coding) together with an interleaver. To simulate the worst situation when coding + interleaving is used, the simulation was realized in Vehicular A channel (Rural Outdoor), mobile's speed $500 \mathrm{~km} / \mathrm{h}$ with channel coherence time $t_{c o h}=0.2 \mathrm{~ms}$.

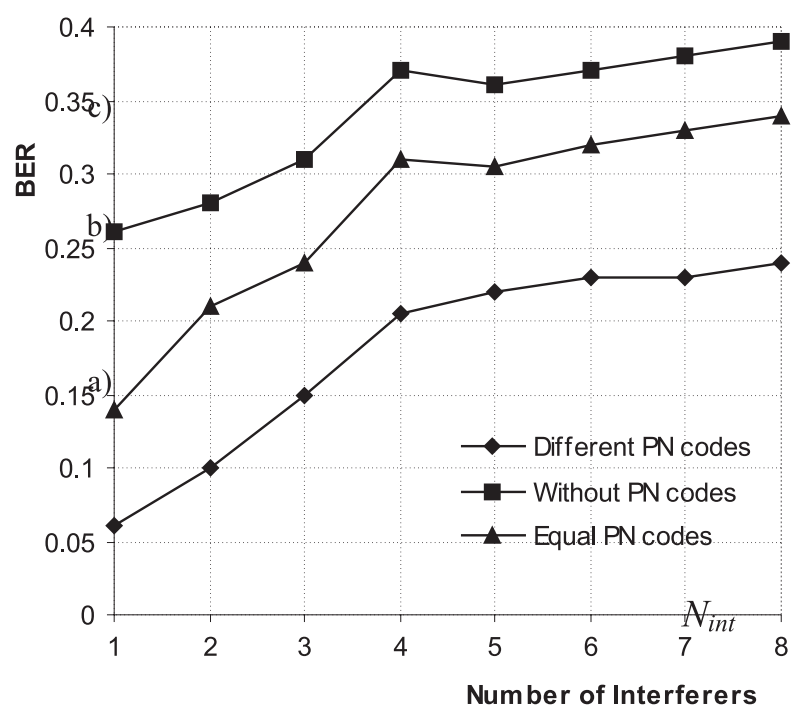

Fig. 6 The dependence of $B E R=f\left(N_{\text {int }}\right)$ for three different situations: a) the system uses different scrambling codes for each mobile, b) the same scrambling code is used in all mobiles in the system, c) spreading without scrambling codes at all

The results show very bad performance of convolutional coding with $\mathrm{R}=1 / 2$ in a situation with very strong interference ( 8 interferers with the same OVSF code). It is obvious that very small spreading factor $(\mathrm{SF}=4)$ in the worst situation is incapable to create required processing gain ensuring the minimum value $E_{b} / I_{0}$ in the receiver.

In addition, the system without convolutional coding and interleaving has better performance than the coded system.

The situation is different in FER simulation (Fig.7b) because the system without coding will have at least one error in each frame $($ FER $=1)$.

To confirm our presumption about insufficient processing gain to suppress mutual interference, the simulation with $\mathrm{SF}=32$ was done (Fig. 8). The advantage of convolutional coding with interleaving is obvious.

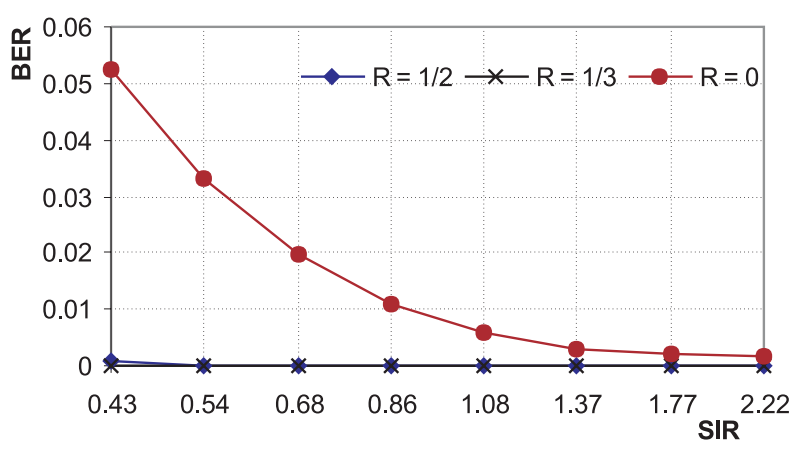

Fig. 8 The dependence $B E R=f(S I R)$ with $S F=32$

The last simulation shows the dependence of BER $=f(\mathrm{SF})$ and $\mathrm{FER}=f(\mathrm{SF})$ for $\mathrm{R}=1 / 2,1 / 3,0$ with $\mathrm{SIR}=0.45$ (const.) (Fig. 9). This situation may arise when all mobiles are at the cell boundary. The poor performance of the convolutional coding with $\mathrm{R}=1 / 2$ up to $\mathrm{SF}=32$ is obvious. On the contrary, the performance of coding with $R=1 / 3$ is poor only up to $S F=16$.

Very promising results of coding with $R=1 / 3$ are shown in Fig. $9 \mathrm{~b}$ where the improvement of performance with the coded system is obvious.

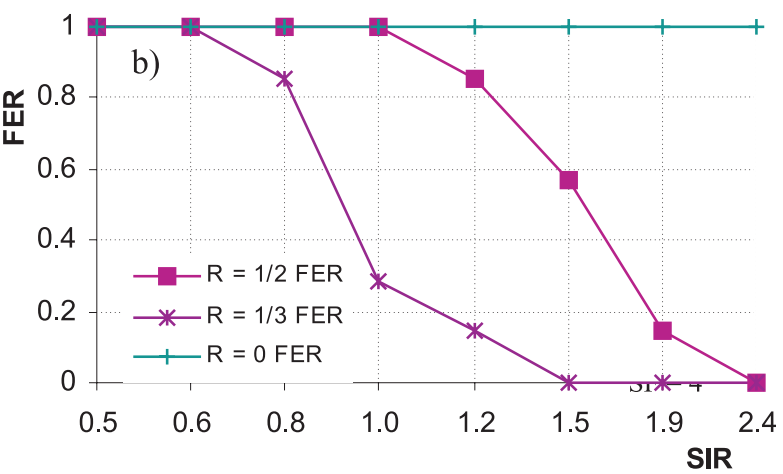

Fig. 7 The dependence a) $B E R=f(S I R)$ and b) $F E R=f(S I R)$ 
a)

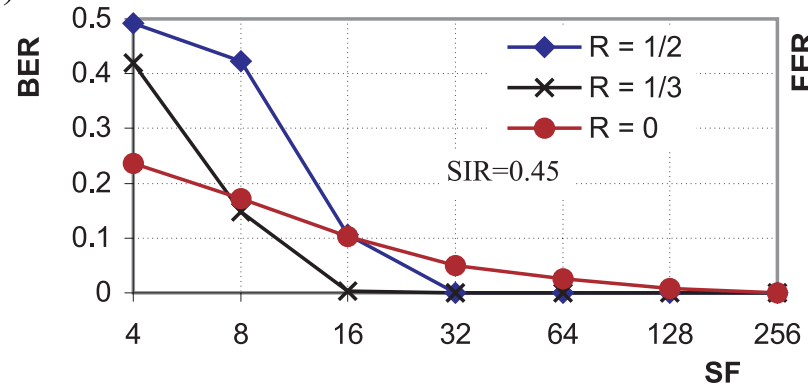

b)

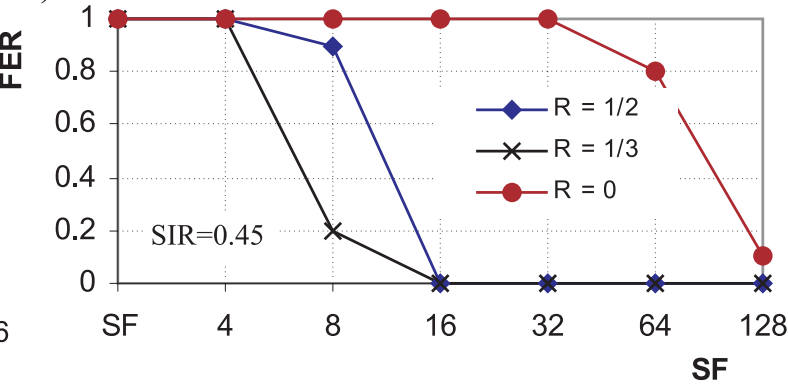

Fig. 7 The dependence a) $B E R=f(S I R)$ and $b) F E R=f($ SIR $)$ Fig. 9 The dependence a) $B E R=f(S F)$ and $b) F E R=f(S F)$

\section{Conclusion}

In the article we presented the uplink UMTS simulator created in MATLAB Simulink with interactive GUI (Graphical User Interface). The simulator allows varying many important simulation parameters of the UMTS system (SF, coding rate, interleaver, parameters of channel and interfering sources, OVSF and scrambling codes, etc.).

The simulations were done for a critical situation with heavy mutual interference in the system. It was shown that convolutional coding with interleaving is not capable of repairing errors when the transmission rate is high $(\mathrm{SF}=4)$.

\section{References}

[1] ROMANO, G., MELIS, B., MASTROFORTI, M.: Link Level Performance of the W-CDMA Radio Interface for UMTS. Rapporti Tecnici CSELT, vol. 28, 2000, pp.421-434. (model CDMASIM).

[2] FAKHRUL, A.: Simulation of third generation CDMA systems. [diploma work], Virginia Polytechnic Institute \& State University, Blacksburg, Virginia, USA, 1999.

[3] MAREK, T., PŠENÁK, V.: Simulácia digitálnych modulácií v mobilnom rádiovom kanáli. [ŠVOČ práca], Žilinská Univerzita, Žilina, 2003.

[4] CASTRO, J. P.: The UMTS network and radio access technology. John Wiley \& Sons, Ltd., England, 2001.

[5] 3GPP TS 25.101: UE Radio transmission and reception (FDD). V6.2.0 (2003-09).

[6] 3GPP TS 25.201: Physical layer - general description. V5.2.0 (2002-09).

[7] 3GPP TS 25.211: Physical channels and mapping of transport channels onto physical channels (FDD). V5.5.0 (2003-09).

[8] 3GPP TS 25.212: Multiplexing and channel coding (FDD). V5.6.0 (2003-09).

[9] 3GPP TS 25.213: Spreading and modulation (FDD). V5.4.0 (2003-09).

[10] 3GPP TR 25.944: Channel coding and multiplexing examples. V4.1.0 (2001-06).

[11] ETSI TR 101 112: Selection procedures for the choice of radio transmission technologies of the UMTS. V3.2.0 (1998-04).

[12] ETSI UMTS XX.07: UTRA FDD; Physical layer procedures. V1.3.1 (1999-02). 